\title{
Ilizarov-Ringfixateur - ein Verfahren zur Kallusdistraktion
}

\author{
Rudolf Vukelic, Cornelius Grimme, Rita Schoop, Klaus Seide, Ulf-Joachim Gerlach, \\ Christian Jürgens
}

\section{Zusammenfassung}

Bei zunehmender Inzidenz der Osteitiden im 21. Jahrhundert und daraus folgenden, zum Teil auch erheblichen, verbleibenden langstreckigen Knochendefekten, besteht nach erfolgreicher Infektsanierung die oberste Priorität in der Rekonstruktion des Knochens, um eine belastbare und voll bewegliche Extremität zu erlangen. Hierfür steht dem erfahrenen Operateur die Kallusdistraktion, basierend auf dem Prinzip der endostalen Osteoneogenese, durch kontinuierliche Distraktion eines sich bildenden Kallus-/Knochengewebes bis zur Ausbildung eines stabilen Röhrenknochens nach vorheriger chirurgischer Durchtrennung (= Kortikotomie/Osteotomie) des betroffenen Knochens im gesunden Areal zur Verfügung. Die Kallusdistraktion hat sich als Standardverfahren bei ausgedehnten Beinverkürzungen sowie langstreckigen Knochendefekten etabliert. Die Behandlung ist aufwendig und komplikationsträchtig und bedarf einer exakten Planung, Operationstechnik sowie sorgfältigen Nachbehandlung.

\section{Ilizarov Ring Fixator: A Procedure for Callus Distraction}

With the increasing incidence of ostitis in the 21st century and the thus resultant, in part also persisting, long bony defects the main priority after management of the infection is to reconstruct the bone in order to achieve a load-bearing and fully mobile limb. For this purpose the method of callus distraction is available for the experienced surgeon; this is based on the principle of endosteal osteoneogenesis through continuous distraction of the forming callus/bone tissue through to formation of a stable hollow bone after previous surgical separation (corticotomy/osteotomy) of the respective bone in a healthy area. Callus distraction is established as the standard procedure for extensive leg shortening and long bony defects. The treatment of tedious and subject to many complications; it thus requires exact planning, a good operative technique and careful postoperative management.

\section{Einleitung}

Der Ilizarov-Ringfixateur hat mittlerweile einen festen Stellenwert in der Behandlung von aussichtslos erscheinenden Knochendefekten. Insbesondere hat sich dieses Verfahren bei beruhigten Infektsituationen mit verbliebenem Knochensubstanzverlust bewährt. Durch eine Kallusdistraktion im Ilizarov-Ringfixateur steht dem erfahrenen Operateur heutzutage eine effektive Methode

OP-JOURNAL 2008; 24: 44-51

(c) Georg Thieme Verlag KG Stuttgart • New York DOI 10.1055/s-2008-1038458

zur Osteoneogenese auch bei schwerwiegender Ausgangssituation, welche früher nicht selten in einer Amputation mündete, zur Verfügung.

Der Ilizarov-Ringfixateur ist ein aus mehreren Ringen beschaffener und um die jeweilige Extremität angelegter Fixateur externe mit gekreuzt gebohrten Olivendrähten oder in seltenen Fällen auch Schanz-Schrauben sowohl proximal als auch distal einer Osteotomiestelle bzw. Fraktur. Die Olivendrähte oder SchanzSchrauben werden gewebeschonend perkutan eingebracht und außerhalb des Hautweichteilmantels am montierten Ringsystem fixiert.
Somit handelt es sich hierbei um ein minimalinvasives Verfahren.

Mit dieser Fixation nach Ilizarov erreicht man nicht nur eine gute sichere Stabilität in 3 Ebenen (Frontal-, Sagittalebene und Rotation), sondern auch gleichzeitig eine dreidimensionale Positionierungsmöglichkeit im Osteotomie-/Frakturbereich. Der Ilizarov-Ringfixateur kann daher zur Achskorrektur, Verlängerung oder auch zur Defektüberbrückung von Extremitäten verwendet werden; in seltenen Fällen auch zur Arthrolyse oder Weichteildehnung.

Der llizarov-Ringfixateur ist ein Fixateur externe mit gekreuzt gebohrten Olivendrähten.

Unter dem Begriff der Kallusdistraktion versteht man die sukzessive Röhrenknochenneubildung über eine äußere oder innere Osteosynthese durch endostales Wachstum nach vorheriger chirurgischer Durchtrennung (=Kortikotomie/ Osteotomie) des betroffenen Knochens im gesunden Areal.

Kallusdistraktion ist die sukzessive Röhrenknochenneubildung durch endostales Wachstum.

\section{Geschichte}

Die ersten Knochenverlängerungen wurden gegen Ende des 19. Jahrhunderts beschrieben. Zunächst erfolgten sie noch unter Einbringung eines Knochenblockes (Hopkins und Penrose, 1889). 1905 führte Codivilla die erste Extremitätenverlängerung ohne eingebrachtes Interponat durch. Er distrahierte intraoperativ den kortikotomierten Oberschenkelknochen durch einmaligen kräftigen Zug über einen eingebrachten Kalkaneusnagel auf die maximal mögliche Länge und fixierte ihn in dieser Position durch Eingipsen. Bei größeren Verlängerungsstrecken musste diese Prozedur mehrmals wiederholt werden. Bier mo- 


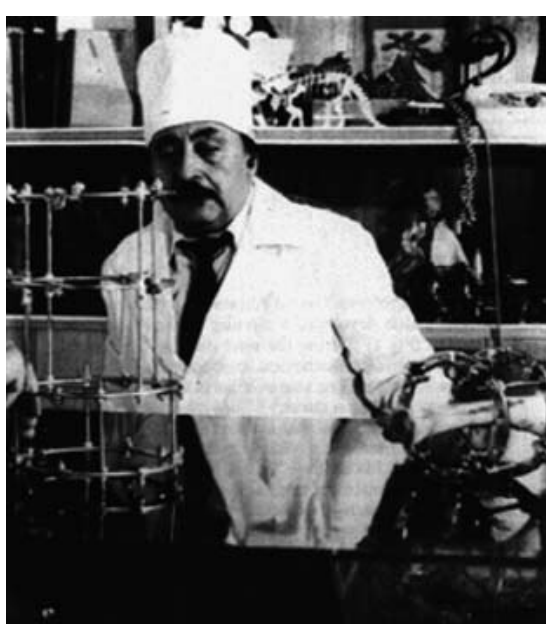

Abb. 1 Prof. Gavril Abramovich llizarov.

difizierte dieses Verfahren 1923 mit primärer Ruhephase von drei bis vier Tagen nach erfolgter Kortikotomie bis zur Ausbildung eines Kallusregenerates aus dem entstandenen Kortikotomiehämatom. Hierdurch erzielte er eine deutliche Abnahme der Knochenheilungsstörung, welche von seinen Vorgängern beschrieben wurde. In den Folgejahren wurden die Verlängerungstechniken durch die Entwicklung von Fixateuren modifiziert, die eine kontrollierte sukzessive Knochendistraktion ermöglichten.

In den frühen Fünfzigern des letzten Jahrhunderts haben parallel und unabhängig voneinander der Österreicher R. Wittmoser und der Russe Gabriel A. Ilizarov (Abb.1) einen Ringfixateur zur Knochenbruchheilung entwickelt. Herrn Wittmoser wurde die Einführung seines Systems durch seinen damaligen Chefarzt Prof. Böhler aus dem Unfallkrankenhaus Webergasse mit den Worten: „Das sieht sehr schön aus aber veröffentlichen Sie es nicht! Man würde es sonst anwenden und es würde ein großes Unglück werden“, verwehrt.

Ilizarov entwickelte seine Methode in Sibirien weiter und behandelte erfolgreich eine große Anzahl von Patienten mit Extremitätenverlängerung. Darüber hinaus betrieb er eine umfangreiche Grundlagenforschung zur Kallusdistraktion im Ringfixateur hinsichtlich der Stabilität und Druckbelastung, welche später in die klinische Routine übernommen wurde.

In der westlichen Welt dauerte die Verbreitung aufgrund politischer Diskrepanzen noch einige Jahre. Erst im November 1980 wurde diese Methode in
Europa durch den Italiener Bianchi-Maiocchi publik gemacht, nachdem dieser von der Ausheilung einer mehrfach voroperierten Osteomyelitis des italienischen Alpinisten Carlo Mauri durch Ilizarov im Ringfixateur mit im Verlauf durchgeführter Kallusdistraktion erfuhr. Daraufhin wurde dieses Verfahren auch in den westlichen Ländern standardisiert und in den Folgejahrzehnten auch in vielen Schwellenländern etabliert.

\section{Indikationen und Behandlungs- konzepte}

Die Kallusdistraktion dient dem Ausgleich von Verkürzungen und Achsfehlstellungen der Extremitätenknochen oder dem Segmenttransport bei Knochendefekten z. B. nach erfolgter Infektsanierung.

Ersteres erfolgt insbesondere nach einer primär notwendig gewordenen Verkürzung im Rahmen der Primärbehandlung offener Frakturen. So ist die primäre Verkürzung und sekundäre Gewebedistraktion eine Behandlungsstrategie bei komplexen Extremitätenverletzungen. Es ist oft günstiger, bei offenen Frakturen mit Defektsituation über eine Distanz von über 3 bis $4 \mathrm{~cm}$ eine primäre Verkürzung und dann frühsekundär nach gesicherter Wundheilung eine Kallusdistraktion durchzuführen. Am häufigsten kommt ein solches Vorgehen heute bei offenen Unterschenkelfrakturen mit entsprechendem Substanzverlust zum Einsatz. Der große Vorteil der Methode besteht darin, dass gleichzeitig mit der gewünschten Längenkorrektur des Knochens auch die Weichteile distrahiert werden können. Somit wird auch der Bedarf an zusätzlichen Rekonstruktionseingriffen minimiert. Darüber hinaus ergibt sich auch kein Risiko einer möglichen Fremdknocheninfektion oder allergischer Reaktionen gegenüber Knochenersatzstoffen.

Unter dem Begriff des Segmenttransportes versteht man den Transport eines Knochensegmentes in einen Defekt, z. B. bei beruhigter Infektsituation, hinein. Hierdurch lassen sich bestehende Knochendefekte bzw. -verluste ausgleichen. Der Segmenttransport erfolgt als longitudinale Kallusdistraktion unter Verschiebung eines intermediären Segmentes (Abb. 2). Der Transport kann sowohl uni- als auch bisegmental erfolgen. Ein solches Vorgehen kommt beispielsweise nach einer radikalen lokalen Resektion avitalen oder nekrotischen Knochens zum Tragen. So können heute Defekte bis $\mathrm{zu} 20 \mathrm{~cm}$ Länge ausgeglichen werden. Bei Defekten unter 3 bis $4 \mathrm{~cm}$ Länge wird dagegen die freie Übertragung von autologer Spongiosa angewandt. Jedoch ist der Segmenttransport der autologen Spongiosaplastik bei Grenzsituationen vorzuziehen, da durch den Segmenttransport im Gegensatz zur Spongiosaplastik ein Röhrenknochen entsteht, welcher deutlich härter ist und bessere elastische Eigenschaften gegenüber Scherund Zugkräften aufweist.

Segmenttransport ist der Transport eines gesunden Knochensegmentes in einen Knochendefekt.

Indikation der Kallusdistraktion:

- Extremitätenverkürzung

- Achsfehlstellung

- Segmenttransport bei Knochendefekt

Defektaufbau mit autologer Spongiosaplastik ist bei Defekten bis zu 3-4cm möglich; bei längeren (bis zu $20 \mathrm{~cm}$ ) ist die Kallusdistraktion vorzuziehen.

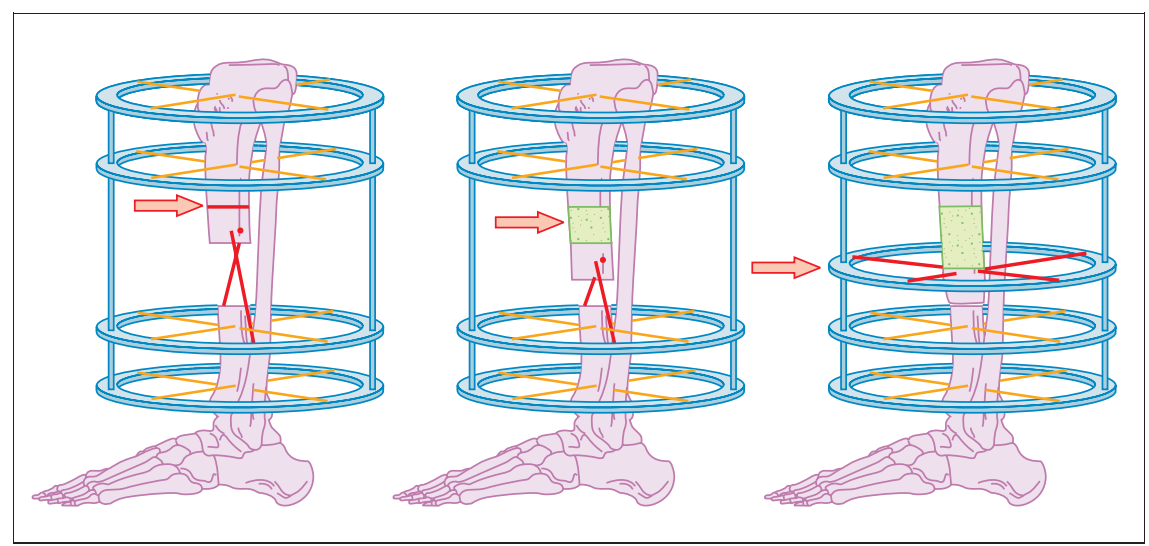

Abb. 2 Schematische Darstellung des Segmenttransports. 


\section{Grundtypen der Kallusdistraktion}

Im Wesentlichen lassen sich die Kallusdistraktionen nach deren Zugrichtung in 3 Grundtypen unterscheiden:

1. Longitudinale Kallusdistraktion

(Längszug)

2. Hemikallusdistraktion

(Zug im Winkel)

3. Laterale Kallusdistraktion

(seitlicher Zug)

Mit der longitudinalen Kallusdistraktion erfolgt die Verlängerung oder der Segmenttransport an den Extremitätenknochen. Zusätzlich kann unter der laufenden Distraktion eine bereits präoperativ bestehende oder unter Distraktion entstandene Achsfehlstellung ohne weiteren operativen Eingriff einfach und schmerzarm für den Patienten im Ilizarov-Ringfixateur korrigiert werden.

Die Hemikallusdistraktion hat ihre Anwendung bei Varus- oder Valgusfehlstellungen. Es wird üblicherweise an der Konkavseite eine inkomplette Kortikotomie durchgeführt, bei erhaltenem Periost an der Konvexseite, und die Osteotomie wie ein Scharnier im Sinne einer Open-wedge-Technik aufgespreizt.

Die laterale Kallusdistraktion dient hierbei nicht zur Knochenverlängerung oder einer Achsfehlstellungskorrektur, sondern zur Knochenverdickung. Am Beispiel des Unterschenkels kann hiermit bei bestehendem Hemidefekt der Tibia mit nur noch einer verbliebenen Kortikalis der Tibia die Fibula in longitudinaler Ebene hemikortikotomiert und zum Defekt herübergezogen werden, um den Defekt zu überbrücken. Jedoch wird diese Variante der Kallusdistraktion eher selten durchgeführt.

Typen der Kallusdistraktion:

- Longitudinale Kallusdistraktion

- Hemikallusdistraktion

- Laterale Kallusdistraktion

\section{Operationsplanung und -technik der Kallusdistraktion über den Ilizarov-Ringfixateur}

Im Rahmen der Indikationsstellung zum Segmenttransport ist im Vorwege abzuklären, zum einem die Gefäß-/Nervensituation der betroffenen Extremität, zum anderen die Compliance des Patienten, da der Segmenttransport ein langwieriges, anspruchvolles Verfahren zum Erhalt der Extremität ist.

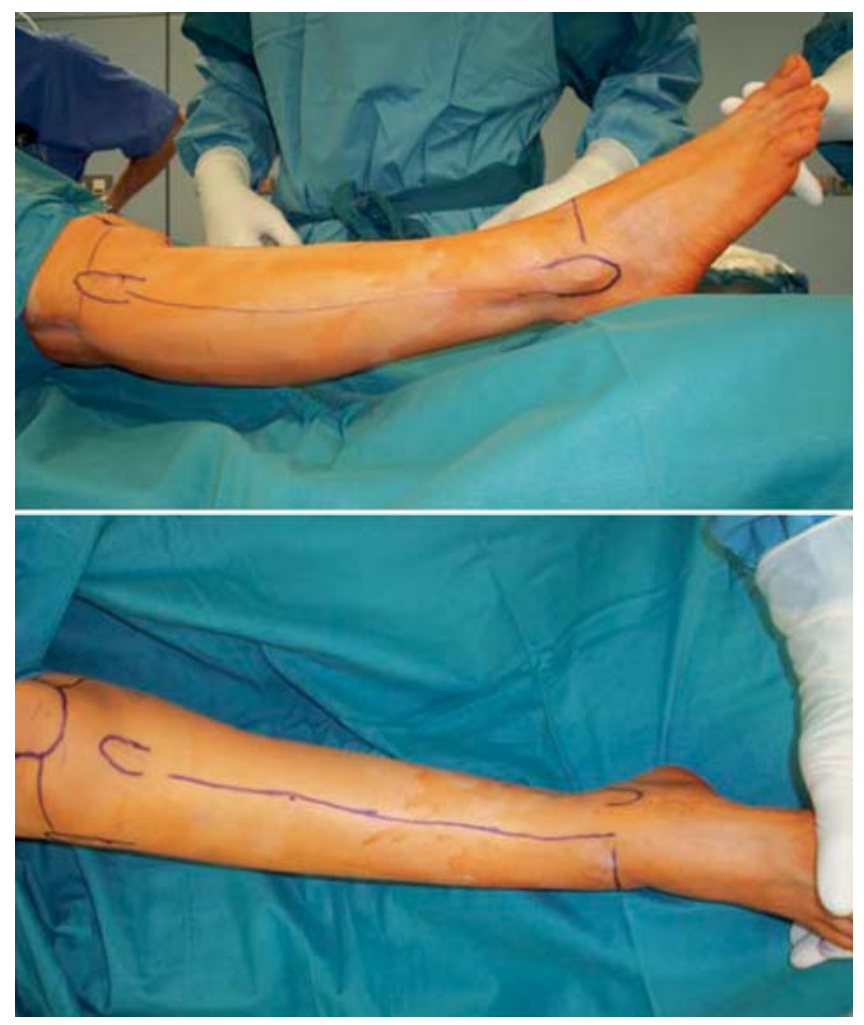

Abb. 3 Anzeichnen der Landmarken.

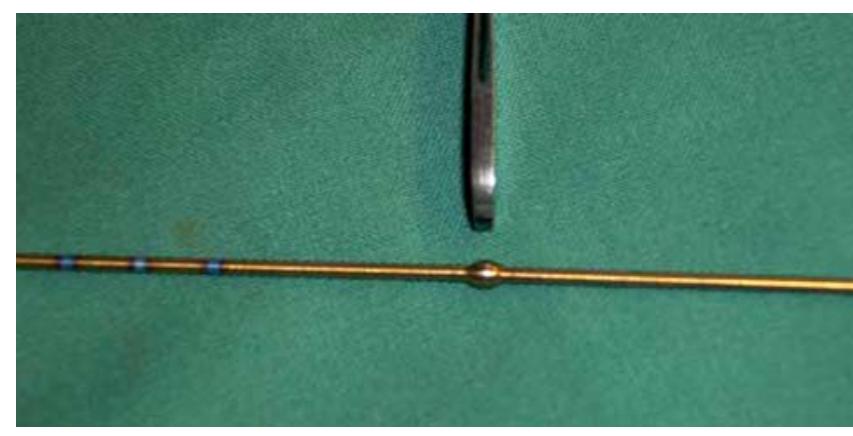

Abb. 4 Olivendraht $=$ Fixations draht.

Nach erfolgter Indikationsstellung und Aufklärung des Patienten beginnt die Operationsplanung mit Überprüfung des Vorhandenseins der ausreichenden Röntgendiagnostik. Es ist empfehlenswert, eine lange Aufnahme mit der Abbildung der gesamten Extremität zu haben. Die Röntgenbilder sollten einen exakten Aufschluss der Größenverhältnisse und Winkel sowie der benachbarten Gelenke wiedergeben. Anhand der nativradiologischen Diagnostik ist präoperativ unter Kenntnis der anatomischen Verhältnisse und der jeweiligen Knochenblutversorgung der Extremität festzulegen, ob der Segmenttransport von proximal nach distal oder umgekehrt erfolgen soll. Zur erleichterten und beschleunigten Operation ist es ggf. sinnvoll, die geplanten Pin-Stellen und das zu transportierende Segment an anatomisch markanten Punkten auf dem Röntgenbild mit einem Fettstift zu markieren.

Die Montage des Ilizarov-Fixateurs erfolgt nach intraoperativem Lagern, Abwaschen und Abdecken des Patienten und vorherigem Anzeichnen von Landmarken wie der unteren Patellafacette, dem medialen und lateralen Kniegelenkspalt, des Fibulaköpfchens, der Tuberositas tibiae, der Tibiavorderkante, des OSG-Spaltes mit distaler Fibula und des Innenknöchels sowie des Knochendefektes mit einem Dermatoscriptor (Abb. 3). Anschließend erfolgt die Auswahl von vier gleich großen Ringen. Hierbei ist es wichtig, darauf zu achten, dass die Ringgröße ventral und ventromedial einen Hautabstand von ca. einem und dorsal bzw. dorsolateral von 
ca. zwei Querfinger hat. Man sollte hierbei lieber zu große als zu kleine Ringe auswählen (Abb.8). Nach der Ringauswahl wird der Ringfixateur auf die Bedürfnisse vormontiert und zur Probe provisorisch an die Extremität angelegt. Hierbei sollte darauf geachtet werden, dass die Beweglichkeit des Kniegelenks nicht wesentlich eingeschränkt und ein minimales Bewegungsausmaß von Extension/Flexion $0-0-90^{\circ}$ gewahrt wird. Erst hiernach erfolgt schrittweise das Einbringen der 2-mm-Drähte. Diese haben einen speziellen Bohrschliff (Bajonettschliff) (Abb.5) und werden mit und ohne Olive gefertigt (Abb.4). Diejenigen ohne Olive werden als Referenzdrähte und diejenigen mit als Fixationsdrähte bezeichnet. Die Drähte gibt es sowohl aus Stahl als auch Titan. In unserem Haus bevorzugen wir die Titandrähte, da diese eine $40 \%$ niedrigere PinInfektionsrate aufweisen.

Landmarken:

- Untere Patellafacette

- Med. und lat. Kniegelenkspalt

- Fibulaköpfchen

- Tuberositas tibiae

- Tibiavorderkante

- OSG-Spalt mit distaler Fibula und Innenknöchel

- Knochendefekt

Zunächst werden die Referenzdrähte in transversaler Ebene sowohl ganz proximal und ganz distal eingebracht. Hierüber kann nun noch eine Achskontrolle und -korrektur erfolgen. Die Referenzdrähte werden mit speziellen Schlitzschrauben am Ringfixateur befestigt. Danach werden die Fixationsdrähte mit den Oliven gegenläufig zueinander in $20-30^{\circ}$ zum Referenzdraht positioniert, wobei die Oliven immer dorsal vom Knochen zum Liegen kommen sollten (Abb.9 und 10). Beim Einbringen der Drähte ist auf die genaue Kenntnis der jeweilig vor Ort befindlichen anatomischen Strukturen zu achten, um eine Verletzung dieser zu vermeiden. Daher sollten die Drähte nur bis zur Durchspießung des Hautweichteilmantels mit dem Bohrer eingebracht und anschließend mit einer Flachzange bis zur endgültigen Position vorgezogen werden. Vor dem Anziehen des Fixationsdrahts mit der Flachzange sollte eine Stichinzision der Haut für die Olive erfolgen. Der Fixationsdraht wird danach bis zum Anschlag der Olive am Knochen angezogen und hiernach mittels Schlitzschraube am Ringfixateur auf der Olivenseite befestigt. Anschließend wird der Fixa-

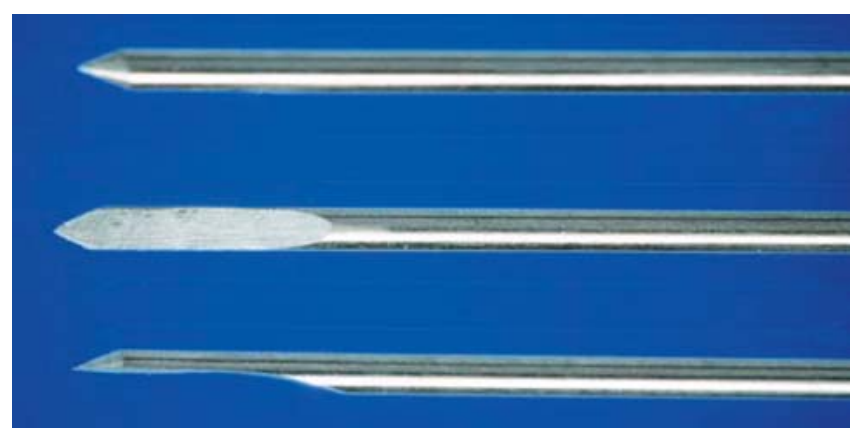

Abb. 5 Bajonettschliff der Drähte.

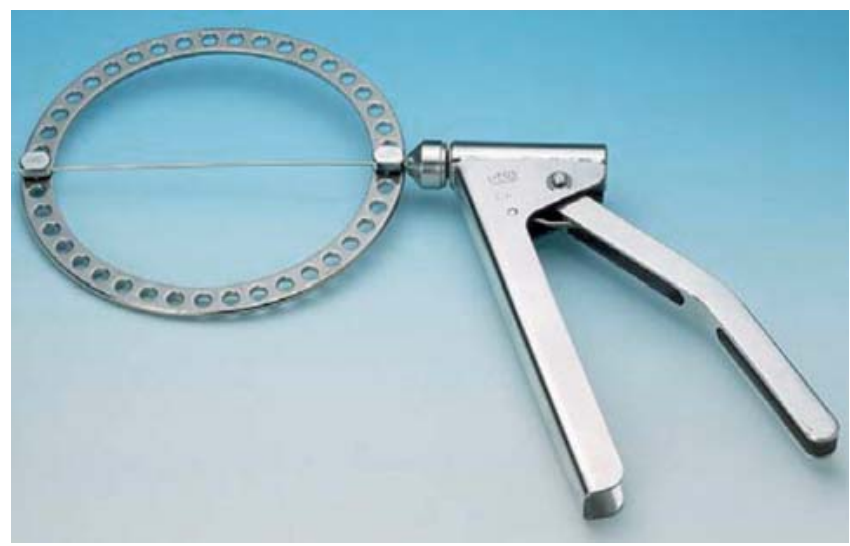

Abb. 6 Spannen des Drahtes auf eine Drahtspannung von ca. $700-1000 \mathrm{Nm}$ mit dem Drahtspanner.

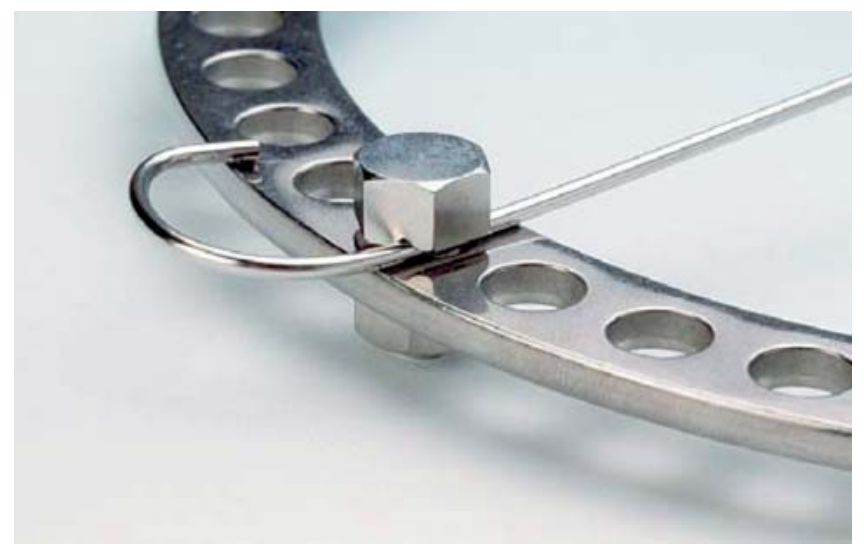

Abb. 7 Fixieren des Drahtes mit einer Schlitzschraube am Ringfixateur.

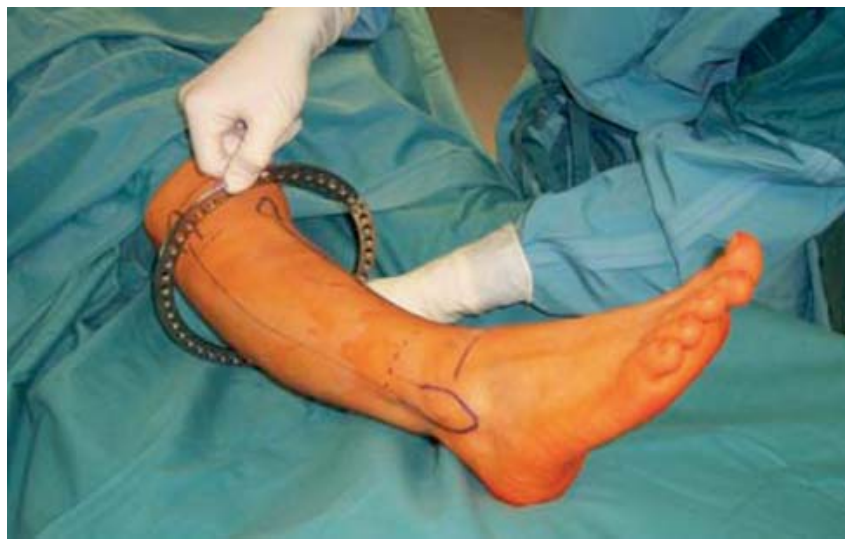

Abb. 8 Anpassen der richtigen Ringgröße an der weitesten Stelle der Extremität. Hier im Bereich der Wade am Beispiel des Unterschenkels. 


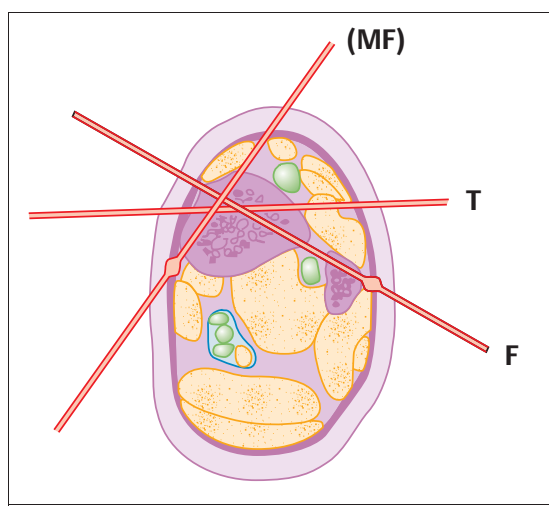

Abb. 9 Schema der Anordnung der Drähte an der proximalen Tibia. $\mathrm{T}=$ transversaler oder Referenzdraht; $F=$ fibularer oder lateraler Draht; MF = medialer Fixationsdraht.

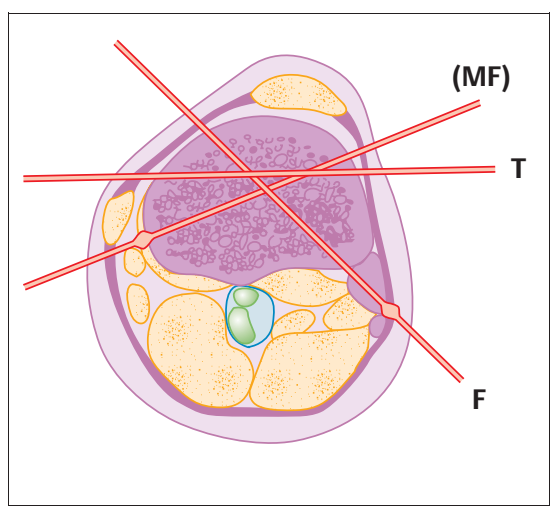

Abb. 10 Schema der Anordnung der Drähte an der distalen Tibia.

tionsdraht mit einer Spannzange auf eine Drahtspannung von ca. 700-1000 $\mathrm{Nm}$ auf der olivenfreien Seite gebracht und unter Einhaltung dieser Spannung ebenfalls mittels Schlitzschraube am Ringfixateur befestigt (Abb.6 und 7). Diese Prozedur wird in gleicher Schrittweise an allen geplanten Ringen durchgeführt bis auf die Referenzdrähte, welche nur proximal und distal verwendet werden (Abb.11 und 12).

Die Fixationsdrähte werden zueinander gegenläufig in $20-30^{\circ}$ zum Referenzdraht positioniert und die Oliven sollten dorsal zum Liegen kommen.

\section{Phasen der Kallusdistraktion}

Als Grundlagen für die Kallusdistraktion als auch den Segmenttransport dienen die Erfahrungen vom Ende des 19. Jahrhunderts und insbesondere die fundierten Forschungsergebnisse von Ilizarov aus den 50er-Jahren.

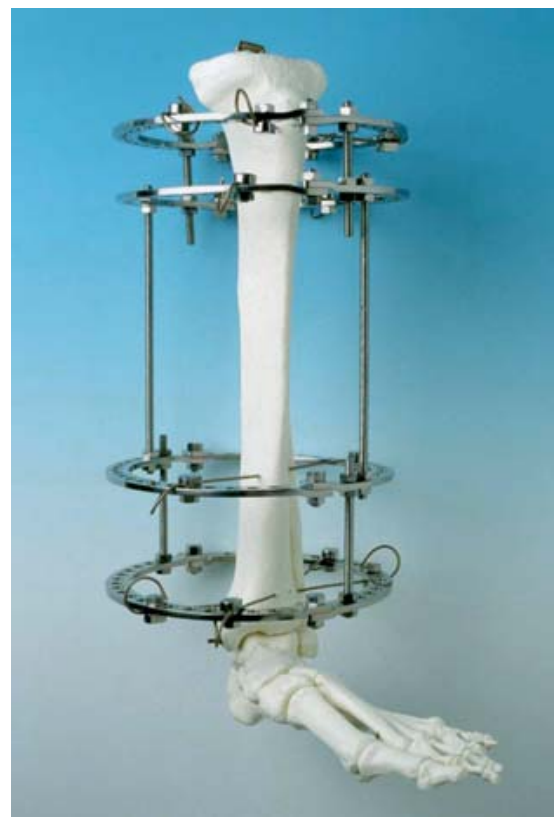

Abb. 11 Schematische Darstellung des angelegten Ilizarov-Ringfixateurs am Unterschenkel.

Ilizarov empfahl eine Kortikotomie mit schonender Durchtrennung des Knochens unter Erhaltung des medullären Gefäßsystems und des Periostschlauches. In neueren Publikationen wird die Notwendigkeit der Erhaltung des inneren Gefäßsystems angezweifelt und die Osteotomie empfohlen. In unserem Hause wird die Kortikotomie nach Ilizarov mit vorherigem fächerförmigen Vorbohren und anschließendem Durchtrennen des Knochens mit einem doppelseitig geschliffenen Ilizarov-Meißel bevorzugt (Abb.13).

Kortikotomie ist die schonende Durchtrennung des Knochens unter Erhalt des medullären Gefäßsystems und $\mathrm{Pe}$ riostschlauchs.

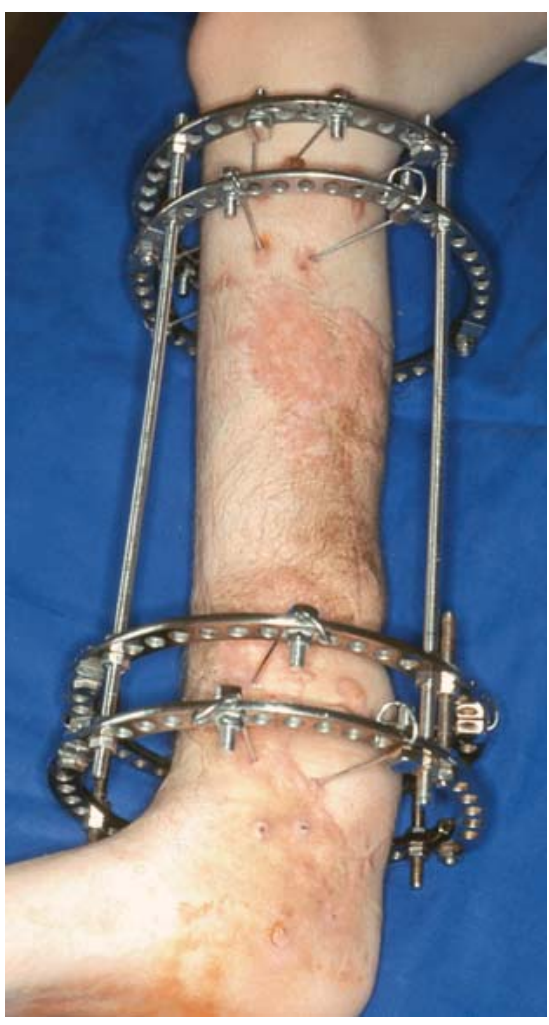

Abb. 12 Klinisches Bild.

Postoperativ halten wir eine von Ilizarov empfohlene Ruhephase von 5 bis 12 Tagen ein, wobei diese Zeit abhängig vom jeweiligen individuellen Krankheitsbild ist. Während dieser Zeit entwickelt sich im metaphysären Frakturspalt ein hochpotentes Fibroblastenregenerat aus dem Kortikotomiehämatom, welches sich später in das gewünschte Kallusgewebe umwandelt. Hiernach beginnt der eigentliche Transport. Es wird mit einer Transportgeschwindigkeit von $4 \times 0,25 \mathrm{~mm}=1 \mathrm{~mm}$ pro Tag gearbeitet. Durch die dabei wirkende Zugspannung und die Druckbelastung auf das Kallusgewebe durch die Faszienlogen entsteht der gewünschte Kallusschlauch. Die ge-

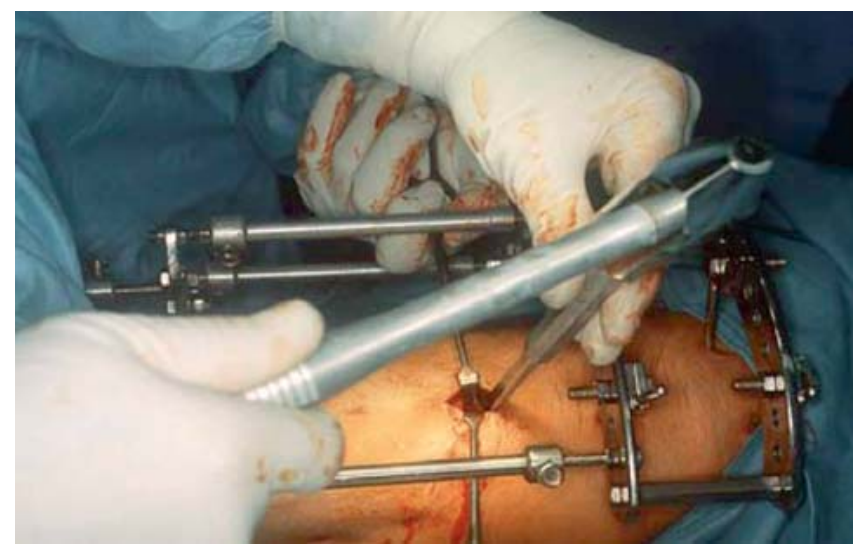

Abb. 13 Intraoperative Kortikotomie mit Ilizarov-Kortikotomiemeißel. 
wöhnliche Zugrichtung ist von proximal nach distal. In seltenen Fällen kann diese aber auch von distal nach proximal erfolgen. Hierbei sollte die Transportgeschwindigkeit nicht mehr als $0,75 \mathrm{~mm}$ pro Tag betragen.

Die Transportgeschwindigkeit beträgt $4 \times 0,25 \mathrm{~mm}=1 \mathrm{~mm}$ pro Tag.

Nach abgeschlossenem Transport folgt die sogenannte Neutralisations- oder Ausreifungsphase. In dieser Phase „reift“ das Kallusgewebe. Die Ausreifung (Mineralisation des Knochens) benötigt etwa die doppelte bis dreifache Zeitdauer der Distraktionszeit. Radiologisch ist dies an einer zunehmenden Mineralisierung erkennbar. Sonografisch lässt sich der wenig mineralisierte Kallus bereits vor der Röntgendarstellung erfassen. Somit stellt die Sonografie in der Frühphase der Distraktion eine Möglichkeit zur Verlaufskontrolle des Knochendurchbaus und der Stellung des Transportsegments dar.

Die Mineralisationsphase benötigt etwa die doppelte bis dreifache Zeitspanne der Distraktionszeit.

Zeigen sich im reifenden Kallus der Distraktionsstrecke Kortikalisstrukturen, kann das Fixationssystem ggf. dynamisiert werden, um die knöcherne Durchbauung zu beschleunigen.

Nach ausreichender Festigung des Kallusgewebes kann das Fixationsystem entfernt werden. Hiermit beginnt die Phase des Remodelings. Der Patient wird typischerweise prophylaktisch, um einen Ermüdungsbruch vorzubeugen, für ca. drei Monate mit einem Brace oder einem teilentlastenden Unterschenkelgehapparat versorgt und sukzessive in Abhängigkeit der Röntgenbefunde und der Schmerzhaftigkeit zur Vollbelastung gebracht.

Der Knochentransport ist oft langwierig. Er erfordert deshalb ein intensives Engagement sowohl vom Patienten als auch Therapeuten. Der Patient ist in der Transportphase regelmäßig zu kontrollieren. Darüber hinaus ist eine frühzeitige intensive physiotherapeutische Behandlung zur Mobilisation angrenzender Gelenke und Aufdehnung des Hautweichteilmantels durchzuführen. Eine Teilbelastung $(10-30 \mathrm{~kg})$ ist dabei für die Ausbildung eines guten Regenerats unabdingbar. Diese wurde von Ilizarov immer wieder betont.
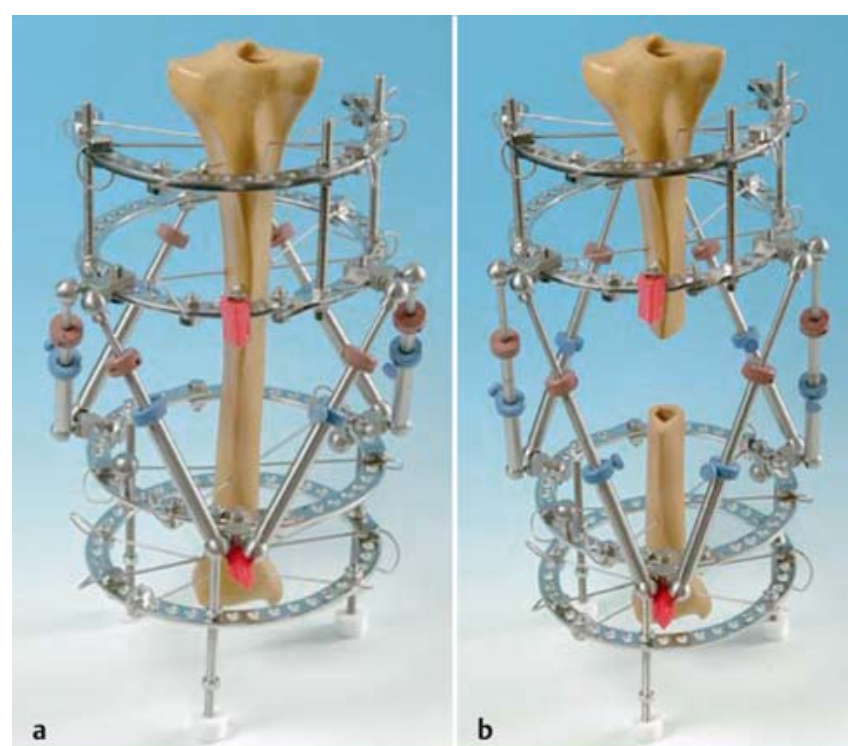

Abb. 14a bis $f$ Hexapod-llizarovFixateur (a). Durch Längeneinstellung der Distraktoren sind beliebige räumliche Bewegungen möglich. Distraktion (b), Translation (c), Torsion (d), Achskorrektur (e). Eine Software ist zur Anwendung erforderlich (f).
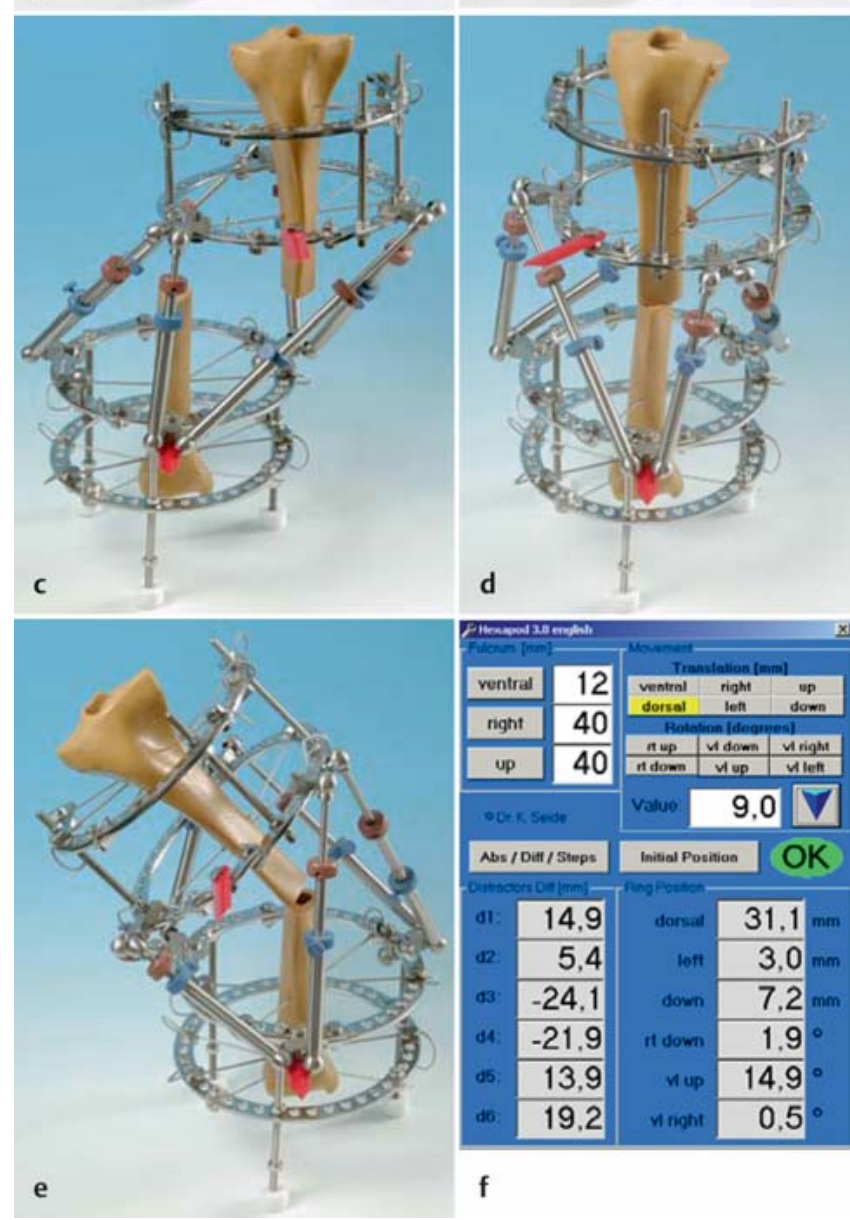

f

\section{Komplikationen}

Ein wesentlicher Vorteil der Kallusdistraktion im Ilizarov-Ringfixateur ist, dass die häufig auftretenden Komplikationen sogenannte Minorkomplikationen sind, die zu den leicht zu behebbaren zählen. Hierunter fallen die PinInfekte, Olivendrahtbrüche, der tempo- räre Nervenschaden, Schmerzen, Beinödeme, Zugdrahtrisse als auch der vorzeitige Durchbau der Distraktionsstrecke. Die Majorkomplikationen sind zum einen Infektion der Distraktionsstrecke und zum anderen intraoperative Gefäß-/ Nervenverletzungen. 
Die Häufigkeit der Komplikationen liegt nach Literaturangabe zwischen 8 und $71 \%$. Dabei dürften Werte zwischen 20 und 50\% für die Minorkomplikationen realistisch sein, wenn man genügend Erfahrung mit dieser Methode hat. Es ist also nicht unwahrscheinlich, dass der einzelne Patient eine der genannten Minorkomplikationen erfährt. Allerdings sind die Komplikationen, wenn sie schnell erkannt und behandelt werden, gut zu beherrschen.

Mit bleibenden Komplikationen ist selten zu rechnen. Genaue Analysen der Komplikationen mit entsprechenden Häufigkeitsangaben sind in der Literatur spärlich.

Minorkomplikationen sind häufig, aber gut beherrschbar. Die Majorkomplikationen sind äußerst selten.

\section{Hexapod}

In den letzten Jahren werden zunehmend Hexapodsysteme verschiedener Hersteller bei der Kallusdistraktion eingesetzt. Der Hexapodmechanismus hat seine Ursprünge in der Robotertechnik und stellt eine kinematische Konstruktion für eine optimale dreidimensionale Positionierung dar. Im Ringfixateur vereinfacht der Hexapodmechanismus vor allem die Korrektur komplexer Fehlstellungen, welche die Hexapoden sukzessive in Kombination mit einer möglichen Kallusdistraktion durchführen. Beim Hexapodfixateur werden die üblicherweise längs verlaufenden Gewindestangen durch sechs über Kugelgelenke an den Ringen fixierte Distraktoren verbunden, welche zirkulär um die Extremität verlaufende Dreiecke darstellen. Die Kugelgelenke sind nicht blockiert, durch Verstellung der Distraktorlängen lassen sich beliebige räumliche Bewegungen realisieren. Eine exakte Positionierung erfordert die Anwendung einer Computersoftware, welche nach Eingabe der Montageparameter sowie der gewünschten Bewegung die entsprechend an den Distraktoren durchzuführenden Einstellungen berechnet. Durch die Anwendung des Hexapoden werden insbesondere Translationen, Rotationen sowie mehrdimensionale Korrekturen (Abb.14) deutlich vereinfacht, da in diesen Fällen die Standardmontagen entweder komplizierte Mechanismen erfordern oder eine sehr exakte Planung von Gelenkpositionen voraussetzen. Die Richtungen der Drehachsen werden im Hexapoden letztlich durch die Software

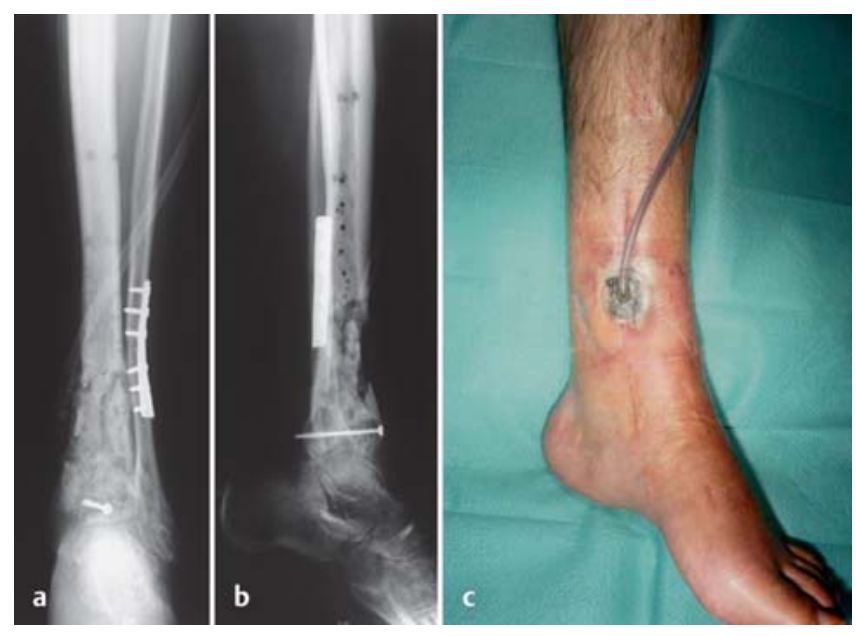

Abb. 15 a bis c Fallbeispiel. Chronisch sequestrierende distale Tibiaosteitis mit OSGEmpyem.

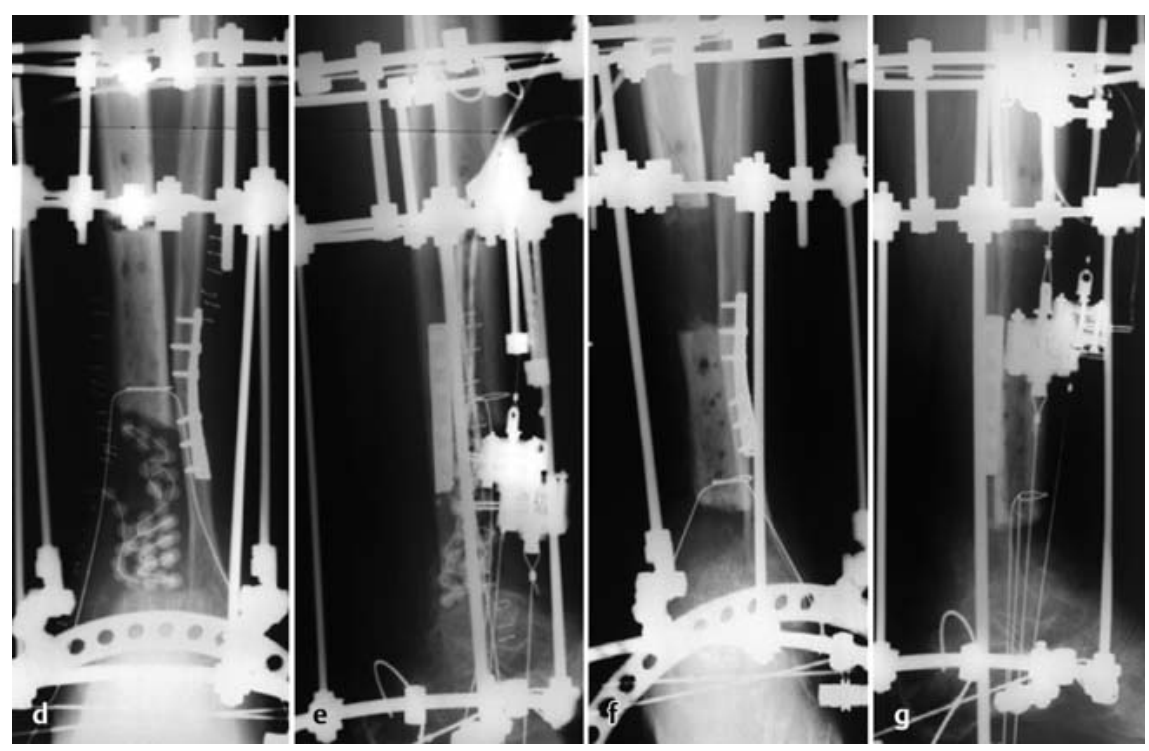

Abb. $15 \mathbf{d}$ bis $\mathbf{g}$ Sequest Irektomie, Septopal-Ketteneinlage und Stabilisierung im Ilizarov-Fixateur $(\mathbf{d}, \mathbf{e})$, Segmenttransport $(\mathbf{f}, \mathbf{g})$.

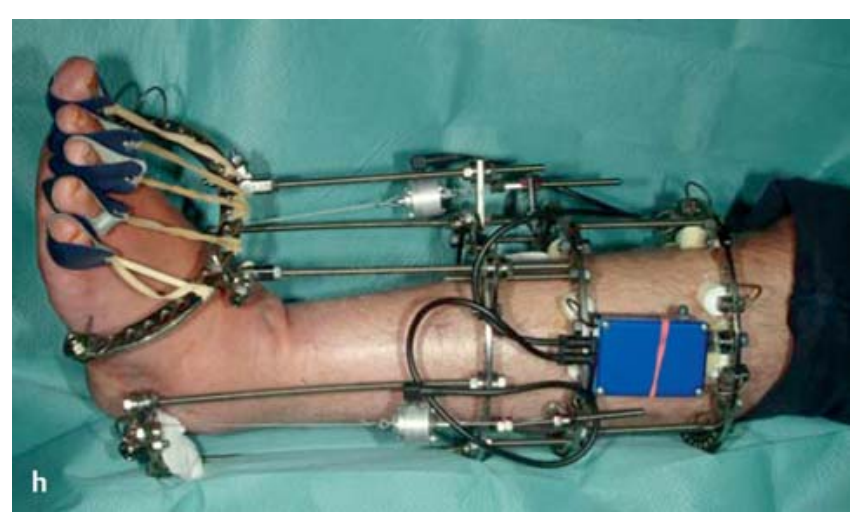

Abb. 15h

Segmenttransport

realisiert und sind somit auch noch während der Behandlung leicht zu modifizieren. Aktuelle weitere Entwicklungen des Hexapoden sind mit Elektromotoren und mechanischen Sensoren versehene Systeme.
Das Hexapodensystem hat die Möglichkeit der dreidimensionalen Positionierung und findet Anwendung bei Translations-, Rotations- und mehrdimensionalen Korrekturen. 


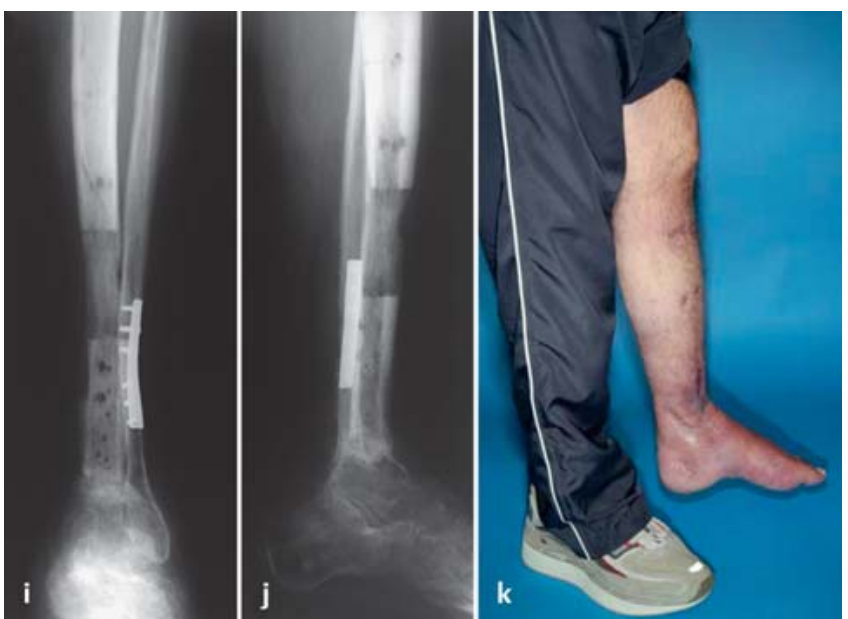

\section{Fallbeispiel}

Ein 62-jähriger Unfallverletzter hatte sich im Rahmen eines Arbeitsunfalls eine geschlossene distale Unterschenkelschaftfraktur links zugezogen. Die primäre Versorgung war mittels Plattenosteosynthese und OSG-übergreifendem Fixateur externe erfolgt. Im Verlauf war es zu einer Wund-ORSA-Infektion gekommen. Es war eine vorzeitige Materialentfernung, ein Wunddébridement und eine Sequestektomie erfolgt. Ein Knochendefekt war mittels autologem kortikospongiösen Spann überbrückt und der verbliebene Hautweichteildefekt mit Vacuseal-Verbänden behandelt worden (Abb.15a bis c). Im weiteren Verlauf war es zu einer Exazerbation des Infektes gekommen.
Abb. 15i bis $\mathbf{k}$ Regenerat $(\mathbf{i}, \mathbf{j})$, klinisches Ergebnis (k).

Bei Aufnahme bestand eine chronische distale Tibiaosteitis mit chronischem OSG-Empyem, sodass eine neuerliche operative Sanierung mit radikaler Sequestektomie, ausführlichem Wunddébridement, Septopal-Ketteneinlage und Stabilisierung im Ilizarov-Ringfixateur notwendig wurde (Abb.15i bis k). Hierdurch kam es zur Beruhigung der Osteitis und des Empyems. Der durch die Operation entstandene langstreckige Knochendefekt $(8,5 \mathrm{~cm})$ wurde nach erfolgter Hautweichteilsanierung mittels Segmenttransport rekonstruiert (Abb.15f bis $\mathbf{h}$ ). Der Unfallverletzte ist nun nach ca. einem halben Jahr nach stattgehabtem Segmenttransport unter Vollbelastung beider unterer Extremitäten in orthopädisch zugerichteten Schuhen mobil (Abb.15d und e).

\section{Literatur}

Ilisarov GA. Transossous Osteosynthesis. Berlin: Springer, 1992

\section{Rudolf Vukelic}

Assistenzarzt UWC

Dr. med. Cornelius Grimme

Leitender Oberarzt, Septische

Knochen- und Gelenkchirurgie

Rita Schoop

Oberärztin, Septische Knochenund Gelenkchirurgie

Priv.-Doz. Dr. med. Klaus Seide

Wissenschaftlicher Leiter

Biomechanik Labor

Dr. med. Ulf-Joachim Gerlach Leitender Arzt, Septische Knochen- und Gelenkchirurgie Prof. Dr. med. Christian Jürgens

Ärztlicher Direktor,

Chefarzt UWC-Abteilung

Abteilung für Unfall- und Wiederherstellungschirurgie Berufsgenossenschaftliches Unfallkrankenhaus Hamburg Bergedorfer Straße 10 21033 Hamburg 